\title{
New fusion protein reduces IL-1-mediated inflammation
}

Alpha-1-antitrypsin (AAT), a naturally occurring inhibitor of serine proteases, has been implicated in caspase-1-independent processing of IL-1 $\beta$ - a major mediator of inflammation in gouty arthritis.

Leo Joosten and colleagues now show that administration of recombinant human AAT fused with an IgG1-Fc fragment (AAT-Fc) suppresses inflammation in a mouse model of gouty arthritis.

Intraperitoneal injection of $50 \mu \mathrm{g}$ AAT-Fc $1 \mathrm{~h}$ prior to induction of gouty arthritis (knee intra-articular injection of monosodium urate crystals plus fatty acid C16.0 [MSU/C16.0]) reduced joint inflammation assessed $4 \mathrm{~h}$ after disease induction (85-91\% reduction compared with control mice treated with bulk

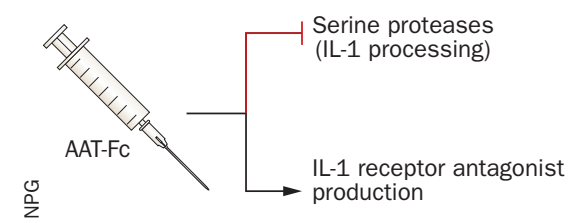

immunoglobulin, $P<0.001)$. Similar results were observed with plasma-derived AAT, although AAT-Fc was effective at concentrations 40-fold lower. Mice treated with AAT-Fc had lower levels of IL- $1 \beta$ and IL-6 in the inflamed synovium $(63 \%$ and $65 \%$ lower, respectively; $P<0.001)$ and reduced immune cell infiltration compared with control mice $(P<0.001)$.

The anti-inflammatory effect of AAT-Fc can be explained, at least partially, by its ability to inhibit serine proteases. "One of the unique findings that was not expected was that AAT-Fc inhibited the processing of the IL- $1 \beta$ precursor by leukocyte proteinase-3 [PR3, also known as myeloblastin], a major neutrophil enzyme known to process the precursor into the active cytokine," explains Joosten. Given that most immune cells infiltrating the synovium in acute gouty arthritis are neutrophils, PR3 inhibition is likely to be a key mechanisms of action of AAT-Fc. Additional experiments confirmed that AAT-Fc inhibited IL- $1 \beta$ release from human monocytes stimulated with MSU/C16.0 (76\% reduction compared with control, $P<0.01)$ and increased the production of IL-1 receptor antagonist -an endogenous inhibitor of the IL-1 receptor-both in vitro (in human cells) and in vivo (in mice).

The use of plasma-derived AAT in humans, for example in patients with acute myocardial infarction, has shown longterm safety, leading the authors to believe that AAT-Fc could be a promising therapy for the treatment of acute gouty arthritis. "In addition to the clinical development of AAT-Fc for acute gout, there are several other inflammatory and immunemediated diseases which we believe can be treated with AAT-Fc," adds Joosten.

\section{João H. Duarte}

Original article Joosten, L. A. B. et al. Alpha-1-antitrypsin-Fc fusion protein ameliorates gouty arthritis by reducing release and extracellular processing of IL- $1 \beta$ and by the induction of endogenous IL-1Ra. Ann. Rheum. Dis. doi:101136/annrheumdis-2014-206966 